\title{
Intravenous Fluid Selection for Unruptured Intracranial Aneurysm Clipping : Balanced Crystalloid versus Normal Saline
}

\author{
Jian Kang, ${ }^{1, *}$ Young Joo Song, ${ }^{1, *}$ Sujeong Jeon, ${ }^{1}$ Junghwa Lee, ${ }^{1}$ Eunsook Lee, Ju-Yeun Lee, ${ }^{2}$ Euni Lee, ${ }^{2}$ Jae Seung Bang, ${ }^{3}$ \\ Si Un Lee, ${ }^{3}$ Moon-Ku Han, ${ }^{4}$ Chang Wan $0 h,{ }^{3}$ Tackeun $\mathrm{Kim}^{3}$ \\ Department of Pharmacy,' Seoul National University Bundang Hospital, Seoungnam, Korea \\ College of Pharmacy \& Research Institute of Pharmaceutical Sciences, ${ }^{2}$ Seoul National University, Seoul, Korea \\ Department of Neurosurgery, ${ }^{3}$ Seoul National University Bundang Hospital, Seoul National University College of Medicine, Seongnam, Korea \\ Department of Neurology, ${ }^{4}$ Seoul National University Bundang Hospital, Seoul National University College of Medicine, Seongnam, Korea
}

Objective : While balanced crystalloid $(B C)$ could be a relevant fluid regimen with buffer system compared with normal saline (NS), there have been no studies on the optimal fluid for surgery of an unruptured intracranial aneurysm (UIA). This study aimed to compare the effects of fluid regimens between NS and BC on the metabolic and clinical outcomes of patients who underwent surgery for UIA.

Methods : This study was designed as a propensity score matched retrospective comparative study and included adult patients who underwent UIA clipping. Patient groups were categorized as NS and BC groups based on the types of pre-operative fluid and the amount of fluid administered during surgery. The primary outcomes were defined as electrolyte imbalance and acidosis immediately after surgery. The secondary outcomes were the length of stay in the intensive care unit (ICU) and duration from the end of the operation to extubation.

Results : A total of 586 patients were enrolled in this study, with each of 293 patients assigned to the NS and BC groups, respectively. Immediately after surgery, serum chloride levels were significantly higher in the NS group. Compared to the NS group, the BC group had lower incidence rates of acidemia (6.5\% vs. $11.6 \%, p=0.043)$ and metabolic acidosis $(0.7 \%$ vs. $4.4 \%, p=0.007)$. As compared to NS group, BC group had significantly shorter duration from the end of the operation to extubation ( $250 \pm 824$ vs. $122 \pm 372$ minutes, $p=0.016)$ and length of stay in ICU (1.37 \pm 1.11 vs. $1.12 \pm 0.61$ days, $p=0.001)$. Throughout multivariable analysis, use of $B C$ was found to be significant factor for favorable post-operative results.

Conclusion : This study showed that the patients who received BC during UIA clipping had lower incidence of metabolic acidosis, earlier extubation and shorter ICU stay compared to those who received NS. Therefore, using BC as a peri-operative fluid can be recommended for patients who undergo surgery for UIA.

Key Words : Balanced crystalloid · Normal saline · Intracranial aneurysm, Unruptured · Crystalloid solutions · Acidosis · Fluid therapy.

- Received : September 4, 2020 •Revised : October 1, 2020 •Accepted : October 20, 2020

- Address for reprints : Tackeun Kim

Department of Neurosurgery, Seoul National University Bundang Hospital, 82 Gumi-ro 173beon-gil, Bundang-gu, Seongnam 13620, Korea

Tel : +82-31-787-7161, Fax : +82-31-787-4059, E-mail : tackeun.kim@snu.ac.kr, ORCID : https://orcid.org/0000-0002-4375-8095

*Jian Kang and Young Joo Song contributed equally to this work.

This is an Open Access article distributed under the terms of the Creative Commons Attribution Non-Commercial License (http://creativecommons.org/licenses/by-nc/4.0) which permits unrestricted non-commercial use, distribution, and reproduction in any medium, provided the original work is properly cited. 


\section{INTRODUCTION}

The incidence of unruptured intracranial aneurysms (UIAs) has exhibited a marked increase owing to an increase in healthcare screenings ${ }^{11,13)}$. Although treatment of UIA is effective in preventing subarachnoid hemorrhage, a tailored decision for the treatment of UIA should be made in consideration of possible complication profiles, because window of the benefit over risk is relatively narrow in UIA treatment, unlike treatment for subarachnoid hemorrhage $e^{6,12)}$. To maximize the risk-benefit ratio of clipping surgery for UIA, surgical and medical complications need to be controlled. Many complementary methods, such as intraoperative neuromonitoring and indocyanine green angiography, have been introduced to reduce the risk of complications during clipping surgery for $\mathrm{UIA}^{4,5,21)}$.

The adequate peri-operative fluid management should be considered, also. The general principle of fluid management for neurosurgical anesthesia is maintaining normovolemia and plasma osmolality to avoid cerebral edema. Hence, normal saline (NS) has been recommended for severe brain inju$\mathrm{ry}^{1,2)}$. However, NS can cause metabolic acidosis and can slightly inhibit kidney function ${ }^{8)}$. Thus, to lower post-operative adverse events in the setting of UIA, post-operative acidosis (POA) and post-operative electrolyte imbalance (POEI) should be considered with NS administration. In a previous meta-analysis, the use of buffered fluids has been associated with less metabolic derangement, in particular hyperchloremia and metabolic acidosis, compared with non-buffered fluids such as $\mathrm{NS}^{3)}$. Another prospective randomized controlled study revealed that balanced crystalloid (BC) provided significantly better control over acid-base balance, sodium and chloride levels with elective neurosurgery ${ }^{7}$. Moreover, NS infusion increased variation in serum chloride compared with BC in children undergoing brain tumor resection ${ }^{14)}$.

As a result, fluid management for UIA clipping has been gradually changed from a NS-based regimen to a buffered BC regimen at our institute. Thus, in this report, we compared the clinical data related with UIA clipping for describing the differences in POA, POEI, and clinical outcomes according to the fluid regimens.

\section{MATERIALS AND METHODS}

This study was approved by Institutional Review Board of Seoul National University Bundang Hospital (IRB No. B-1909-562-109) and adhered to the recommendations of the Declaration of Helsinki for biomedical research involving human subjects. This retrospective cohort study included adult patients (18 years of age or older) who underwent UIA clipping at our institute between April 1, 2003 and July 31, 2019.

The inclusion and exclusion criteria are summarized in Fig. 1. Patients administered with fluids other than NS or BC were excluded. The NS group consisted of patients administered with NS as pre-operative fluid and administered with NS in an amount greater than $50 \%$ of the total fluid volume during surgery. The same criteria were applied to the BC group. The fluid administered to the $\mathrm{BC}$ group was a single product (Plasma Solution-A, HK inno.N Corp., Seoul, Korea) containing sodium chloride (5.26 g), sodium gluconate (5.02 g), sodium acetate hydrate (3.68 g), potassium chloride (0.37 g), and magnesium chloride $(0.3 \mathrm{~g})$ in $1000 \mathrm{~mL}$ of fluid. Propensity score matching was performed to minimize selection bias.

We collected the following data through electronic medical records and a clinical data warehouse system, which was a big data querying platform. The classification of aneurysms targeted for surgery is as follows. Anterior cerebral artery (ACA) aneurysms were defined as aneurysms arose from the ACA, including the anterior communicating artery. Internal carotid artery (ICA) aneurysms included the posterior communicating artery aneurysms, the anterior choroidal artery aneurysms and other aneurysms arose along the ICA. Middle cerebral artery (MCA) aneurysms were all kind of aneurysms belonged to the MCA and bifurcation. Posterior circulation aneurysms were defined as all aneurysm arose from the posterior circulations.

To compare POEI and POA, the laboratory results of arterial blood gas analysis and serum electrolytes were recorded immediately after surgery and on post-operative day 1 . Through this study, acidemia was defined as an arterial blood $\mathrm{pH}<7.35$. Metabolic acidosis was defined as a serum bicarbonate concentration $<22 \mathrm{mEq} / \mathrm{L}$ with acidemia. For analysis of clinical outcomes, we collected information on the length of stay in the intensive care unit (ICU) (in days) and the duration from the end of the operation to extubation (in minutes). Throughout the study period, extubation had been conducted under 
same criteria (Glasgow coma score $\geq 13$, patient able to initiate an inspiratory effort, and normal $\mathrm{PaO}_{2}$ without ventilator support).

Age, sex, body mass index (BMI), hypertension, diabetes mellitus, and pre-operative glomerular filtration rate (GFR) were recorded to control potential confounding factors. Factors related with surgery (operation time, total fluid volume and transfusion volume administered) were also investigated.

All statistical analyses were performed using $\mathrm{R}$ software (version 3.5.3; The R Foundation for Statistical Computing,
Vienna, Austria). Continuous variables are presented as mean \pm standard deviation and were analyzed by an independent ttest. The chi-square test was used to compare categorical variables. Variables showed statistical significance through univariable analysis were selected for multivariable analysis. Binomial logistic regression was performed to measure the association between incidence rates of acidemia, metabolic acidosis, and other variables. The goodness of fit and the simplicity of the model was evaluated using Akaike information criterion (AIC). For the continuous outcome variable, multi-

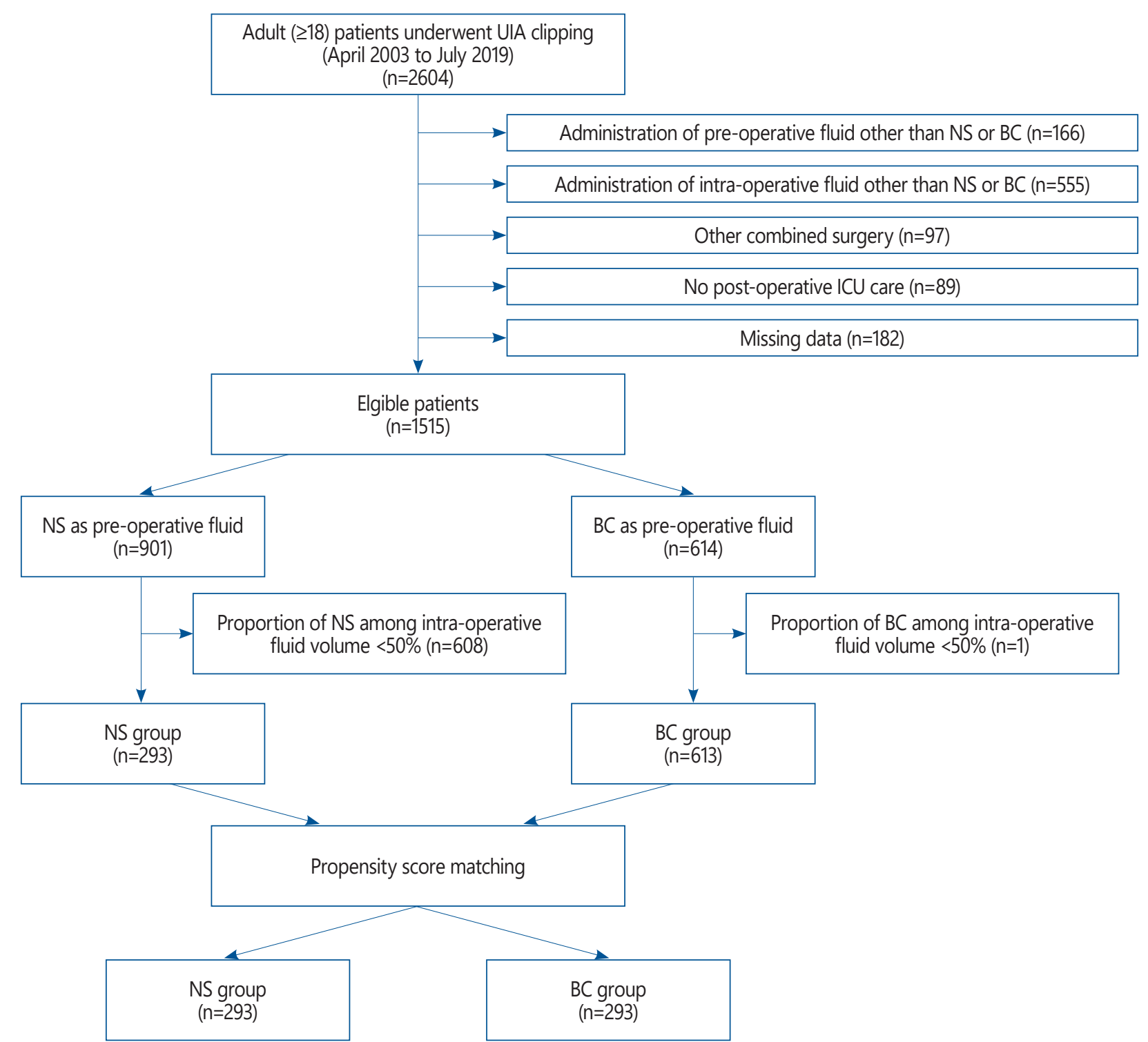

Fig. 1. Flowchart shows enrollment of study subjects. Following the inclusion and exclusion criteria, a total 906 patients (293 NS group and 613 BC group) were matched by propensity score. As a result, 293 patients are allocated to each group. UIA : unruptured intracranial aneurysm, NS : normal saline, BC : balanced crystalloid, ICU : intensive care unit. 
variable linear regression was used to analyze the linear coefficients of variables using bi-directional variable selection based on AIC. With each final model, additional fitting was conducted with variables showing multicollinearity and their interactions. $p$-values $<0.05$ were regarded as statistically significant.

\section{RESULTS}

A total of 906 patients met the inclusion criteria : 293 and

Table 1. Patients characteristics

\begin{tabular}{|c|c|c|c|}
\hline & $\begin{array}{l}\text { NS group } \\
(n=293)\end{array}$ & $\begin{array}{c}\text { BC group } \\
(n=293)\end{array}$ & $p$-value \\
\hline Age (years) & $56.5 \pm 9.7$ & $56.9 \pm 10.2$ & 0.660 \\
\hline Female sex & $194(66.2)$ & $198(67.6)$ & 0.792 \\
\hline $\mathrm{BMI}\left(\mathrm{kg} / \mathrm{m}^{2}\right)$ & $25.0 \pm 3.3$ & $24.8 \pm 3.6$ & 0.616 \\
\hline Sodium (mmol/L) & $140.8 \pm 2.3$ & $140.8 \pm 2.2$ & 0.971 \\
\hline Potassium (mmol/L) & $4.20 \pm 0.39$ & $4.20 \pm 0.38$ & 0.966 \\
\hline Chloride (mmol/L) & $103.8 \pm 2.4$ & $104.1 \pm 2.4$ & 0.197 \\
\hline $\mathrm{GFR}\left(\mathrm{mL} / \mathrm{min} / 1.73 \mathrm{~m}^{2}\right)$ & $91.0 \pm 31.5$ & $93.0 \pm 24.7$ & 0.481 \\
\hline Hypertension & $155(52.9)$ & $158(53.9)$ & 0.868 \\
\hline Diabetes & $26(8.9)$ & $31(10.6)$ & 0.577 \\
\hline Operation time (minutes) & $240.9 \pm 86.4$ & $241.0 \pm 109.7$ & 0.996 \\
\hline Total fluid volume (mL) & $1699 \pm 619$ & $1683 \pm 852$ & 0.799 \\
\hline Total transfusion volume (mL) & $215 \pm 444$ & $164 \pm 447$ & 0.167 \\
\hline \multicolumn{4}{|l|}{ Location of aneurysms } \\
\hline MCA & $144(49.1)$ & $157(53.6)$ & \\
\hline ACA & $75(25.6)$ & $62(21.2)$ & \\
\hline $\mathrm{ICA}$ & $34(11.6)$ & $40(13.7)$ & \\
\hline POST & $1(0.3)$ & $4(1.4)$ & \\
\hline $\mathrm{ACA}+\mathrm{MCA}$ & $16(5.5)$ & $15(5.1)$ & \\
\hline $\mathrm{ICA}+\mathrm{MCA}$ & $11(3.8)$ & $4(1.4)$ & \\
\hline$A C A+I C A$ & $7(2.4)$ & $7(2.4)$ & \\
\hline MCA+POST & $2(0.7)$ & $2(0.7)$ & \\
\hline ICA+POST & $1(0.3)$ & $0(0.0)$ & \\
\hline$A C A+I C A+M C A$ & $2(0.7)$ & $0(0.0)$ & \\
\hline $\mathrm{ACA}+\mathrm{MCA}+\mathrm{POST}$ & $0(0.0)$ & $1(0.3)$ & \\
\hline ICA+MCA+POST & $0(0.0)$ & $1(0.3)$ & \\
\hline
\end{tabular}

Values are presented as mean \pm standard deviation or number (\%). NS : normal saline, BC : balanced crystalloid, BMI : body mass index, GFR : glomerular filtration rate, MCA : middle cerebral artery, ACA : anterior cerebral artery, ICA : internal carotid artery, POST : posterior circulation
613 patients of NS and BC group, respectively. Throughout propensity score matching with age, sex, BMI, pre-operative measurement of serum levels of sodium, potassium, chloride, GFR, history of hypertension, diabetes mellitus, operation time and the amount of total volume of fluid and transfusion, 293 patients could be allocated to each group. The basic characteristics of patients and distribution of aneurysms are summarized in Table 1. The two groups showed a similar distributions of all variables used in matching process.

Table 2 shows the results of the laboratory tests immediately after surgery. The serum levels of sodium and potassium did not show statistical differences, however, the mean serum chloride level of the NS group $(109.1 \pm 2.9 \mathrm{mmol} / \mathrm{L})$ was significantly higher than that of the BC group $(107.8 \pm 3.1 \mathrm{mmol} / \mathrm{L})$ $(p<0.001)$. The arterial blood gas analysis results showed that the arterial $\mathrm{pH}$ was significantly lower in the NS group (7.39 \pm $0.04)$ than in the $\mathrm{BC}$ group $(7.42 \pm 0.05, p<0.001) . \mathrm{HCO}_{3}^{-}$level was also significantly lower in the NS group compared to that of the BC group ( $22.7 \pm 1.8$ vs. $24.1 \pm 2.1, p<0.001)$. On the other hand, partial pressure of carbon dioxide $\left(\mathrm{pCO}_{2}\right)$ showed similar results. There were three patients in the $\mathrm{BC}$ group and zero

Table 2. Immediate post-operative laboratory tests and clinical outcomes by fluid types

\begin{tabular}{|c|c|c|c|}
\hline & $\begin{array}{l}\text { NS group } \\
(n=293)\end{array}$ & $\begin{array}{c}\text { BC group } \\
(n=293)\end{array}$ & $p$-value \\
\hline Sodium (mmol/L) & $142.4 \pm 2.4$ & $142.5 \pm 2.6$ & 0.476 \\
\hline Potassium (mmol/L) & $3.91 \pm 0.33$ & $3.96 \pm 0.32$ & 0.070 \\
\hline Chloride (mmol/L) & $109.1 \pm 2.9$ & $107.8 \pm 3.1$ & $<0.001^{*}$ \\
\hline $\mathrm{GFR}\left(\mathrm{mL} / \mathrm{min} / 1.73 \mathrm{~m}^{2}\right)$ & $112.9 \pm 45.7$ & $111.9 \pm 28.9$ & 0.763 \\
\hline $\mathrm{pH}$ & $7.39 \pm 0.04$ & $7.42 \pm 0.05$ & $<0.001^{*}$ \\
\hline $\mathrm{pCO}_{2}(\mathrm{mmHg})$ & $37.9 \pm 4.9$ & $37.8 \pm 6.3$ & 0.836 \\
\hline $\mathrm{HCO}_{3}^{-}(\mathrm{mmol} / \mathrm{L})$ & $22.7 \pm 1.8$ & $24.1 \pm 2.1$ & $<0.001^{*}$ \\
\hline Anion gap (mmol/L) & $10.51 \pm 2.23$ & $10.74 \pm 2.40$ & 0.244 \\
\hline Base excess (mmol/L) & $-1.67 \pm 1.78$ & $-0.05 \pm 2.35$ & $<0.001^{*}$ \\
\hline Acidemia & $34(11.6)$ & $19(6.5)$ & $0.043^{*}$ \\
\hline Metabolic acidosis & $13(4.4)$ & $2(0.7)$ & $0.007^{*}$ \\
\hline Vasopressor & $0(0.0)$ & $3(1.0)$ & 0.249 \\
\hline Length of stay in ICU (days) & $1.37 \pm 1.11$ & $1.12 \pm 0.61$ & $0.001^{*}$ \\
\hline $\begin{array}{l}\text { Duration from the end of the } \\
\text { operation to extubation (minutes) }\end{array}$ & $250 \pm 824$ & $122 \pm 372$ & $0.016^{*}$ \\
\hline
\end{tabular}

Values are presented as mean \pm standard deviation or number (\%). *Indicates statistical significance $(p<0.05)$. NS : normal saline, BC: balanced crystalloid, GFR : glomerular filtration rate, ICU : intensive care unit 
patients in the NS group who required the administration of vasopressor after surgery, but there was no statistical difference. The incidence of acidemia was significantly higher in the NS group compared to the BC group (11.6\% vs. $6.5 \%$, $p=0.043)$. The rate of metabolic acidosis was also significantly higher in the NS group compared to the BC group (4.4\% vs. $0.7 \%, p=0.007$ ). An analysis of the clinical outcomes showed that the length of stay in the ICU was significantly shorter among patients in the $\mathrm{BC}$ group $(1.37 \pm 1.11$ days) compared to those in the NS group $(1.12 \pm 0.61$ days, $p=0.001)$. With regards to the mean duration from the end of the operation to extubation, the NS group required twice as much time (250 \pm 824 minutes) compared to the BC group (122 \pm 372 minutes) ( $p=0.016$ ).

The binomial logistic regression analysis showed that BMI, operation time, total fluid volume and fluid type were significantly related with development of acidemia. Throughout multivariable analysis, interaction variable between operation

Table 3. Binomial logistic regression for immediate post-operative acidemia and metabolic acidosis

\begin{tabular}{|c|c|c|c|c|c|c|}
\hline & \multicolumn{3}{|c|}{ Univariable analysis } & \multicolumn{3}{|c|}{ Multivariable analysis } \\
\hline & OR & $95 \% \mathrm{Cl}$ & $p$-value & OR & $95 \% \mathrm{Cl}$ & $p$-value \\
\hline \multicolumn{7}{|l|}{ Outcome : acidemia } \\
\hline Age & 0.99 & $0.95-1.03$ & 0.574 & & & \\
\hline Sex (female) & 0.98 & $0.51-1.81$ & 0.957 & & & \\
\hline $\mathrm{BMI}\left(\mathrm{kg} / \mathrm{m}^{2}\right)$ & 1.14 & $1.04-1.25$ & $0.007^{*}$ & 1.12 & $1.03-1.21$ & $0.006^{*}$ \\
\hline Pre-OP sodium (mmol/L) & 0.92 & $0.79-1.06$ & 0.242 & & & \\
\hline Pre-OP potassium (mmol/L) & 0.94 & $0.44-2.03$ & 0.874 & & & \\
\hline Pre-OP chloride (mmol/L) & 1.11 & $0.96-1.27$ & 0.152 & & & \\
\hline Pre-OP GFR (mL/min/1.73 m²) & 1 & $0.98-1.01$ & 0.480 & & & \\
\hline Hypertension & 1.05 & $0.55-1.97$ & 0.891 & & & \\
\hline Diabetes & 0.76 & $0.27-2.13$ & 0.596 & & & \\
\hline Operation time (minutes) & 1 & $1.00-1.01$ & $0.035^{*}$ & & Eliminated & \\
\hline Total fluid volume $(\mathrm{mL})$ & 1 & $1.00-1.00$ & $0.006^{*}$ & & Eliminated & \\
\hline Total transfusion volume (mL) & 1 & $1.00-1.00$ & 0.950 & & & \\
\hline NS group & 1.96 & $1.07-3.58$ & $0.030^{*}$ & 1.9 & $1.05-3.44$ & $0.033^{*}$ \\
\hline \multicolumn{7}{|l|}{ Outcome : metabolic acidosis } \\
\hline Age & 0.91 & $0.84-0.98$ & $0.011^{*}$ & & Eliminated & \\
\hline Sex (female) & 2.73 & $0.59-12.70$ & 0.200 & & & \\
\hline $\mathrm{BMI}\left(\mathrm{kg} / \mathrm{m}^{2}\right)$ & 1.23 & $1.01-1.49$ & $0.036^{*}$ & & Eliminated & \\
\hline Pre-OP sodium (mmol/L) & 0.87 & $0.66-1.15$ & 0.321 & & & \\
\hline Pre-OP potassium (mmol/L) & 0.89 & $0.21-3.82$ & 0.870 & & & \\
\hline Pre-OP chloride (mmol/L) & 1.17 & $0.92-1.50$ & 0.210 & & & \\
\hline Pre-OP GFR (mL/min/1.73 m²) & 0.95 & $0.92-0.98$ & $0.003^{*}$ & 0.97 & $0.95-0.99$ & $0.009^{*}$ \\
\hline Hypertension & 1.08 & $0.30-3.90$ & 0.908 & & & \\
\hline Diabetes & 0.72 & $0.08-6.76$ & 0.771 & & & \\
\hline Operation time (minutes) & 1.01 & $1.00-1.01$ & 0.106 & & & \\
\hline Total fluid volume $(\mathrm{mL})$ & 1 & $1.00-1.00$ & 0.263 & & & \\
\hline Total transfusion volume (mL) & 1 & $1.00-1.00$ & 0.529 & & & \\
\hline NS group & 5.16 & $1.060-25.06$ & $0.042^{*}$ & 6.14 & $1.36-27.70$ & $0.018^{*}$ \\
\hline
\end{tabular}

*Indicates statistical significance ( $p<0.05)$. OR: odds ratio, $\mathrm{Cl}$ : confidence interval, BMI : body mass index, OP : operation, GFR: glomerular filtration rate, NS : normal saline 
time and the total fluid volume was added and evaluated. Throughout additional fitting, operation time, total fluid volume and their interaction variable were eliminated from the final model. According to the final model, BMI of patients (adjusted odds ratio $[\mathrm{OR}], 1.12$; 95\% confidence interval [CI], $1.03-1.21 ; p=0.006)$ and the use of NS for fluid management (adjusted OR, 1.90; 95\% CI, 1.05-3.44; $p=0.033$ ) were significant predictors for the development of acidemia.

With regards to metabolic acidosis, age, BMI, pre-operative GFR and fluid type were statistically significant variables by univariable analysis. Interactions were found between BMI and pre-operative GFR, and between age and pre-operative GFR. Using two second-ordered interaction variables among them, age, BMI and interaction variables were eliminated from the final model. According to the final model, pre-operative GFR (adjusted OR, 0.97; 95\% CI, 0.95-0.99; $p=0.009$ ) and the use of NS for fluid management (adjusted OR, 6.14; 95\% CI, 1.36-27.70; $p=0.018)$ were significantly associated (Table 3).

The factors affecting the duration from the end of the oper-

Table 4. Linear regression for clinical outcomes

\begin{tabular}{|c|c|c|c|c|}
\hline & \multicolumn{2}{|c|}{ Univariable analysis } & \multicolumn{2}{|c|}{ Multivariable analysis } \\
\hline & Coefficient \pm SE & $p$-value & Coefficient \pm SE & $p$-value \\
\hline \multicolumn{5}{|c|}{ Outcome : duration from end of operation to extubation (minutes) } \\
\hline Age & $-0.753 \pm 3.284$ & 0.819 & & \\
\hline Sex (female) & $18.7 \pm 53.6$ & 0.726 & & \\
\hline $\mathrm{BMI}\left(\mathrm{kg} / \mathrm{m}^{2}\right)$ & $9.73 \pm 8.13$ & 0.232 & & \\
\hline Pre-OP sodium (mmol/L) & $10.7 \pm 12.0$ & 0.376 & & \\
\hline Pre-OP potassium (mmol/L) & $26.3 \pm 63.0$ & 0.676 & & \\
\hline Pre-OP chloride (mmol/L) & $-5.19 \pm 11.44$ & 0.650 & & \\
\hline Pre-OP GFR (mL/min/1.73 m²) & $-1.09 \pm 1.25$ & 0.385 & & \\
\hline Hypertension & $77.3 \pm 51.8$ & 0.136 & & \\
\hline Diabetes & $-145 \pm 82$ & 0.078 & & \\
\hline Operation time (minutes) & $-0.397 \pm 0.368$ & 0.280 & & \\
\hline Total fluid volume (mL) & $0.0731 \pm 0.0491$ & 0.137 & & \\
\hline Total transfusion volume $(\mathrm{mL})$ & $0.651 \pm 0.065$ & $<0.001^{*}$ & $0.655 \pm 0.053$ & $<0.001^{*}$ \\
\hline NS group & $95.1 \pm 47.4$ & $0.045^{*}$ & $99.8 \pm 47.4$ & $0.036^{*}$ \\
\hline \multicolumn{5}{|c|}{ Outcome : length of stay in ICU (days) } \\
\hline Age & $0.00136 \pm 0.00463$ & 0.768 & & \\
\hline Sex (female) & $-0.0957 \pm 0.0764$ & 0.211 & & \\
\hline $\mathrm{BMI}\left(\mathrm{kg} / \mathrm{m}^{2}\right)$ & $0.00885 \pm 0.01149$ & 0.442 & & \\
\hline Pre-OP sodium (mmol/L) & $0.00861 \pm 0.01716$ & 0.616 & & \\
\hline Pre-OP potassium (mmol/L) & $0.0225 \pm 0.0894$ & 0.802 & & \\
\hline Pre-OP chloride (mmol/L) & $-0.00797 \pm 0.01629$ & 0.625 & & \\
\hline Pre-OP GFR (mL/min/1.73 m²) & $-0.00246 \pm 0.00165$ & 0.136 & & \\
\hline Hypertension & $-0.0707 \pm 0.0734$ & 0.335 & & \\
\hline Diabetes & $0.0861 \pm 0.1178$ & 0.465 & & \\
\hline Operation time (minutes) & $0.00124 \pm 0.00052$ & $0.016^{*}$ & $0.00154 \pm 0.00039$ & $<0.001^{*}$ \\
\hline Total fluid volume (mL) & $0.0000718 \pm 0.0000695$ & 0.302 & & \\
\hline Total transfusion volume $(\mathrm{mL})$ & $0.000608 \pm 0.000092$ & $<0.001^{*}$ & $0.000627 \pm 0.0000867$ & $<0.001^{*}$ \\
\hline NS group & $0.210 \pm 0.068$ & $0.002^{*}$ & $0.217 \pm 0.067$ & $0.001^{*}$ \\
\hline
\end{tabular}

*Indicates statistical significance $(p<0.05)$. SE : standard error, BMI : body mass index, OP : operation, NS : normal saline, ICU : intensive care unit 
ation to extubation and the length of stay in the ICU are summarized in Table 4 with the results from the linear regression. By univariable analysis for extubation time, total transfusion volume and fluid type were selected for multivariable regression. As there was no collinearity between two variables, the final model was fitted to use total transfusion volume and fluid type as a predictive variable for extubation time. The use of NS resulted delay of 99.8 minutes of extubation time. The $\mathrm{R}^{2}$ of this model was 0.22 (95\% CI, 0.16-0.28).

As to length of stay in ICU, operation time, total transfusion volume and fluid type showed statistical significance by univariable linear regression. Because there was strong linear correlation between operation time and total transfusion volume, further fitting including interaction variable resulted in the elimination of only interaction variable, leaving each variable related. According to the final model, the use of NS, a longer operation time and a larger total transfusion volume were significantly correlated with a longer stay in the ICU. The use of NS resulted delay of 0.2 days of ICU stay. The $\mathrm{R}^{2}$ of this model was 0.19 (95\% CI, 0.14-0.25).

\section{DISCUSSION}

In this study, the use of $\mathrm{BC}$ as a management fluid during surgery for UIA was related with a lower occurrence of POEI and POA. Furthermore, duration from the end of the operation to extubation and ICU stay were significantly shorter among patients in the BC group compared to those in the NS group.

Although there had been no reports regarding the effects of using BC instead of NS during surgery for UIA, the superiority of BC has been reported in critically ill adults when comparing clinical results such as mortality, hospital days, and renal function ${ }^{3,9)}$. Similar to previous reports, the use of BC rather than NS also resulted in a shorter length of stay in the ICU in this study. These clinical findings might be due to the substances and buffer system making up the fluid and the resulting metabolic acidosis. Several reports corroborated our findings : the use of BC was superior to that of NS, especially when comparing serum $\mathrm{pH}$ and chloride levels ${ }^{3,8)}$. A previous study showed that the major determinant of $[\mathrm{H}+]$ was the strong ion difference in the body ${ }^{22)}$. Changes in $[\mathrm{Cl}-]$, which is a major anionic contributor, affects changes in $[\mathrm{H}+]$ homeo- stasis. The development of significant hyperchloremia with large volumes of NS for fluid resuscitation would result in a decrease in strong ion differences and metabolic acidosis ${ }^{16)}$. Hyperchloremic metabolic acidosis is a pathological state that results from bicarbonate loss, rather than acid production or retention. The major $\mathrm{pH}$ buffer system in the human body is the bicarbonate/carbon dioxide $\left(\mathrm{HCO}_{3}{ }^{-} / \mathrm{CO}_{2}\right)$ chemical equilibrium system. A decrease in $\mathrm{HCO}_{3}{ }^{-}$will shift the acid-base balance towards acidic. The pulmonary system regulates $\mathrm{CO}_{2}$ levels through respiration; this condition increases the respiratory rate as the body attempts to decrease $\mathrm{CO}_{2}$ to compensate and then delays the time to extubation ${ }^{18,20)}$. In this study, those who received $\mathrm{BC}$ required approximately half as much time from the end of the operation to extubation compared to those who received NS.

Neurological manifestations, headache, lethargy, stupor, and coma in most severe cases can result from metabolic derangement. Additionally, during periods of profound acidosis, cerebral hypoxia may occur. Signs and symptoms of raised intracranial pressure occur less often when the acidosis is secondary to a metabolic cause rather than a respiratory cause, as alluded to previously, because the development of respiratory acidosis is often more acute ${ }^{24)}$. Therefore, our findings indicating that NS was six times more likely to trigger metabolic acidosis provided important clinical insights for caring of patients who underwent neurologic surgery, as metabolic acidosis was considered as a risk factor potentially leading to poor clinical course in these patients.

Also, some studies found the development of moderate hyperchloremia to be associated with increased in-hospital mortality in patients who experienced an intracerebral hemorrhage $^{19)}$. For those who were hyperchloremic during ICU admission, every $5 \mathrm{mmol} / \mathrm{L}$ increase in chloride was associated with a $37 \%$ increase in odds for hospital mortality. This can be explained by the fact that hyperchloremic metabolic acidosis has been linked to an increased rate of infection via a theoretical augmented neutrophil response and a proinflammatory imbalance ${ }^{17)}$. Infection has been associated with increased hospital mortality as well as worse 3-month outcomes in patients who experienced intracerebral hemorrhage ${ }^{15)}$. Other studies have demonstrated a possible hypocoagulable state and the need for a large volume of blood products after administration of sodium chloride-based fluids during surgical procedures $^{23)}$. In addition, one study found that BC therapy 
was more effective in maintaining normal potassium level and reducing the trend toward hyperchloremic metabolic acidosis than NS therapy during prolonged fluid therapy post-operatively in patients treated for traumatic brain injury ${ }^{10)}$. Similar to these studies, hyperchloremic metabolic acidosis could affect the clinical outcomes of patients in the neurocritical ICU.

This study had some limitations. This was a retrospective study, and the data were derived from a single center, which could not completely exclude a selection bias. Furthermore, the two groups might be not fully comparable due to chronological differences. Thus, we performed propensity score matching to reduce the bias. As a result, statistical differences were not observed between two groups indicating that comparative groups were secured with a certain degree of homogeneity. Furthermore, compared to previous studies that analyzed the effects of fluid regimens in critically ill patients who had various primary diagnoses, one strength of our study was the focus on patients who underwent UIA clipping; therefore, relatively homogeneous characteristics of the patients group could show better comparable effects of fluid management in POEI, POA, and clinical outcomes. Still, further prospective randomized clinical trials are needed to determine a more solid recommendation for the selection of fluid regimen during surgery for UIA.

\section{CONCLUSION}

BC resulted in lower serum chloride ion levels, lower incidence of metabolic acidosis, and shorter duration of intubation and ICU stay. Therefore, using BC can be recommended as an appropriate fluid than NS for managing patients who undergo surgery for UIA in the pre- and intra-operative periods.

\section{CONFLICTS OF INTEREST}

No potential conflict of interest relevant to this article was reported.

\section{INFORMED CONSENT}

This type of study does not require informed consent.

\section{AUTHOR CONTRIBUTIONS}

\author{
Conceptualization : JK, YJS, TK \\ Data curation : JK, YJS \\ Formal analysis : JK, YJS, TK \\ Funding acquisition : TK \\ Methodology : TK \\ Project administration : TK \\ Visualization : TK \\ Writing - original draft : JK, YJS \\ Writing - review \& editing: SJ, JL, EL, JYL, EL, JSB, SUL, \\ $\mathrm{MKH}, \mathrm{CWO}, \mathrm{TK}$
}

\section{ORCID}

$\begin{array}{ll}\text { Jian Kang } & \text { https://orcid.org/0000-0002-5064-2111 } \\ \text { Young Joo Song } & \text { https://orcid.org/0000-0002-7914-2591 } \\ \text { Sujeong Jeon } & \text { https://orcid.org/0000-0001-7497-6776 } \\ \text { Junghwa Lee } & \text { https://orcid.org/0000-0003-4620-2815 } \\ \text { Eunsook Lee } & \text { https://orcid.org/0000-0002-6640-4820 } \\ \text { Ju-Yeun Lee } & \text { https://orcid.org/0000-0002-2261-7330 } \\ \text { Euni Lee } & \text { https://orcid.org/0000-0002-4487-4450 } \\ \text { Jae Seung Bang } & \text { https://orcid.org/0000-0001-9543-8758 } \\ \text { Si Un Lee } & \text { https://orcid.org/0000-0001-5788-2014 } \\ \text { Moon-Ku Han } & \text { https://orcid.org/0000-0003-0166-387X } \\ \text { Chang Wan Oh } & \text { https://orcid.org/0000-0001-7311-7072 } \\ \text { Tackeun Kim } & \text { https://orcid.org/0000-0002-4375-8095 }\end{array}$

\section{- Acknowledgements}

This study was supported by grant No. 14-2018-023 from the Seoul National University Bundang Hospital Research Fund.

\section{References}

1. Bederson JB, Connolly ES Jr, Batjer HH, Dacey RG, Dion JE, Diringer MN, et al. : Guidelines for the management of aneurysmal subarachnoid 
hemorrhage: a statement for healthcare professionals from a special writing group of the Stroke Council, American Heart Association.

Stroke 40 : 994-1025, 2009

2. Brain Trauma Foundation; American Association of Neurological Surgeons; Congress of Neurological Surgeons : Guidelines for the management of severe traumatic brain injury. J Neurotrauma 24 Suppl 1 : S1-S106, 2007

3. Burdett E, Dushianthan A, Bennett-Guerrero E, Cro S, Gan TJ, Grocott $M P$, et al. : Perioperative buffered versus non-buffered fluid administration for surgery in adults. Cochrane Database Syst Rev 12 : CD004089, 2012

4. Byoun HS, Bang JS, Oh CW, Kwon OK, Hwang G, Han JH, et al. : The incidence of and risk factors for ischemic complications after microsurgical clipping of unruptured middle cerebral artery aneurysms and the efficacy of intraoperative monitoring of somatosensory evoked potentials: a retrospective study. Clin Neurol Neurosurg 151 : 128-135, 2016

5. Byoun HS, Oh CW, Kwon OK, Lee SU, Ban SP, Kim SH, et al. : Intraoperative neuromonitoring during microsurgical clipping for unruptured anterior choroidal artery aneurysm. Clin Neurol Neurosurg 186 : 105503, 2019

6. Foreman PM, Ogilvy CS : Unruptured intracranial aneurysms: whom to treat? World Neurosurg $122: 311-312,2019$

7. Hafizah M, Liu CY, Ooi JS : Normal saline versus balanced-salt solution as intravenous fluid therapy during neurosurgery: effects on acid-base balance and electrolytes. J Neurosurg Sci 61 : 263-270, 2017

8. Hahn RG : Should anaesthetists stop infusing isotonic saline? Br J Anaesth $112: 4-6,2014$

9. Hammond DA, Lam SW, Rech MA, Smith MN, Westrick J, Trivedi AP, et al. : Balanced crystalloids versus saline in critically ill adults: a systematic review and meta-analysis. Ann Pharmacother 54 : 5-13, 2020

10. Hassan MH, Hassan WMNW, Zaini RHM, Shukeri WFWM, Abidin HZ, Eu CS : Balanced fluid versus saline-based fluid in post-operative severe traumatic brain injury patients: acid-base and electrolytes assessment. Malays J Med Sci 24 : 83-93, 2017

11. Kim T, Lee H, Ahn S, Kwon OK, Bang JS, Hwang G, et al. : Incidence and risk factors of intracranial aneurysm: a national cohort study in Korea. Int J Stroke 11 : 917-927, 2016

12. Kim YD, Bang JS, Lee SU, Jeong WJ, Kwon OK, Ban SP, et al. : Long-term outcomes of treatment for unruptured intracranial aneurysms in South
Korea: clipping versus coiling. J Neurointerv Surg 10 : 1218-1222, 2018

13. Lee SU, Kim T, Kwon OK, Bang JS, Ban SP, Byoun HS, et al. : Trends in the incidence and treatment of cerebrovascular diseases in Korea : part I. Intracranial aneurysm, intracerebral hemorrhage, and arteriovenous malformation. J Korean Neurosurg Soc 63 : 56-68, 2020

14. Lima MF, Neville IS, Cavalheiro S, Bourguignon DC, Pelosi P, Malbouisson LMS : Balanced crystalloids versus saline for perioperative intravenous fluid administration in children undergoing neurosurgery: a randomized clinical trial. J Neurosurg Anesthesiol 31 : 30-35, 2019

15. Lord AS, Langefeld CD, Sekar P, Moomaw CJ, Badjatia N, Vashkevich A, et al. : Infection after intracerebral hemorrhage: risk factors and association with outcomes in the ethnic/racial variations of intracerebral hemorrhage study. Stroke 45 : 3535-3542, 2014

16. Miller $L R$, Waters $J H$, Provost $C$ : Mechanism of hyperchloremic metabolic acidosis. Anesthesiology 84 : 482-483, 1996

17. Neyra JA, Canepa-Escaro F, Li X, Manllo J, Adams-Huet B, Yee J, et al. : Association of hyperchloremia with hospital mortality in critically III septic patients. Crit Care Med 43 : 1938-1944, 2015

18. Reddi BA : Why is saline so acidic (and does it really matter?). Int J Med Sci $10: 747-750,2013$

19. Riha HM, Erdman MJ, Vandigo JE, Kimmons LA, Goyal N, Davidson $\mathrm{KE}$, et al. : Impact of moderate hyperchloremia on clinical outcomes in intracerebral hemorrhage patients treated with continuous infusion hypertonic saline: a pilot study. Crit Care Med 45 : e947-e953, 2017

20. Sharma S, Hashmi MF, Aggarwal S: Hyperchloremic acidosis. Treasure Island: StatPearls Publishing, 2021

21. Son YJ, Kim JE, Park SB, Lee SH, Chung YS, Yang HJ : Quantitative analysis of intraoperative indocyanine green video angiography in aneurysm surgery. J Cerebrovasc Endovasc Neurosurg 15 : 76-84, 2013

22. Stewart PA : Modern quantitative acid-base chemistry. Can J Physiol Pharmacol 61 : 1444-1461, 1983

23. Waters JH, Gottlieb A, Schoenwald P, Popovich MJ, Sprung J, Nelson DR : Normal saline versus lactated Ringer's solution for intraoperative fluid management in patients undergoing abdominal aortic aneurysm repair: an outcome study. Anesth Analg 93 : 817-822, 2001

24. Yee $A H$, Rabinstein $A A$ : Neurologic presentations of acid-base imbalance, electrolyte abnormalities, and endocrine emergencies. Neurol Clin 28 : 1-16, 2010 